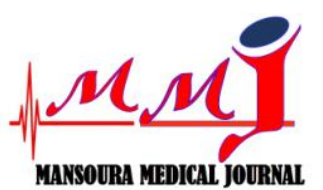

Since 1987 ISSN: 1110-211X

\author{
Mansoura Medical Journal \\ (Official Journal of Mansoura Faculty of Medicine) \\ pISSN: 1110-211X; eISSN: 2735-3990
}

\title{
Assessment of Attention Impairment in ADHD children
}

\author{
Hazem Alawadli' ${ }^{1}$, Omayma Afsah ${ }^{2}$, Essam A. A. Elmagd ${ }^{3}$, Tamer Abou-Elsaad ${ }^{2}$ \\ ${ }^{1}$ Phoniatric Unit, ORL Department, Faculty of Medicine, Aswan University, Egypt. \\ ${ }^{2}$ Phoniatric Unit, ORL Department, Faculty of Medicine, Mansoura University, Egypt. \\ ${ }^{3}$ ORL Department, Faculty of Medicine, Aswan University, Egypt.
}

DOI: $10.21608 / \mathrm{mjmu} .2021 .98058 .1041$

Submit Date: 2021-09-26

Accept Date: $2021-10-10$

Available online:

\section{Keywords}

- $\mathrm{ADHD}$

- Attention Assessment

- Attention disorders

- Attention deficit

- Attention impairment

\section{Abstract}

Attention develops throughout childhood and enables adaptive behavior for meeting daily demands in school and during social interactions. Attention deficit hyperactivity disorder (ADHD) represents the most common cause of childhood attention impairment. Arriving at an accurate diagnosis is often the central goal of assessment, and in clinical settings, there is additional emphasis on the need for assessment to aid in case conceptualization, treatment planning, and progress monitoring. Assessment of ADHD requires parents, teachers and clinical interviews, observation, neuropsychological measures and neurophysiological investigations. Rating scales may be the most useful diagnostic tool in demonstrating attention impairment, but their utility is limited as such measures do not demonstrate particular impairments. So, objective measures of attention may be needed to detect more subtle forms of impairment in children with ADHD. The purpose of the current review was to describe the different assessment tools of attention impairment in ADHD children in order to identify the most effective methods of assessment to provide accurate diagnosis and treatment planning.

Corresponding author: Hazem Alawadli, MSc., Phoniatric Unit, ORL Department, Aswan University, Aswan, 81528, Egypt. E-mail:dr.hazemsami@gmail.com. Mobile: 00201001184330 


\section{INTRODUCTION}

Attention processes are the essential aspect of daily intellectual performance and many cognitive functions, like perception, learning, memory, and information processing. Also, they are important for the acquisition of reading, spelling, and calculating [1]. Attention develops throughout childhood and enables adaptive behavior for meeting daily demands in school and during social interactions [2].

Although attention deficit hyperactivity disorder (ADHD) represents the most common cause of childhood attention impairment [3]. Attention disorder may be observed in children with acquired or neurological disorders like acquired brain injuries, leukaemia, Autistic spectrum disorder (ASD), depression, epilepsy, Tourette syndrome, and Turner syndrome [4].

Arriving at an accurate diagnosis is often the central goal of assessment, and in clinical settings, there is additional emphasis on the need for assessment to aid in case conceptualization, treatment planning, and progress monitoring [5].

Clinical interviews, questionnaires, and performance-based measures (or their combination) are common methods to assess ADHD core and accessory symptoms [6]. Rating scales may be the most useful diagnostic tool in demonstrating attention impairment, but their utility is limited as such measures do not demonstrate particular impairments. So, objective measures of attention may be needed to detect more subtle forms of impairment in children with ADHD.

The purpose of the current review was to describe the different assessment tools of attention impairment in ADHD children in order to identify the most effective methods of assessment to provide accurate diagnosis and treatment planning.

\section{Assessment of ADHD in children}

\subsection{Subjective measures:}

\section{A. DSM Criteria for diagnosis}

Based on the DSM V-criteria, there are three presentations of ADHD:

a. Predominantly inattentive presentation presents with symptoms including being easily distracted, forgetful, daydreaming, disorganization, poor concentration, and difficulty completing tasks.

b. Predominantly hyperactive/impulsive presentation presents with excessive fidgetiness and restlessness, hyperactivity, difficulty waiting and remaining seated, immature behavior; destructive behaviors may also be present.

c. Combined presentation is a combination of the two other presentations.

This subdivision is based on presence of at least six out of nine long-term (lasting at least six months) symptoms of inattention, hyperactivityimpulsivity, or both. To be considered, the symptoms must have appeared by the age of six to twelve and occur in more than one environment (e.g. at home and at school or work). The symptoms must be inappropriate for a child age and there must be evidence that it is causing social, school or work related problems [3].

Most ADHD preschoolers present with the combined ADHD subtype. The predominantly hyperactive impulsive subtype is more common in preschoolers than in older children, as hyperactivity tends to decrease with age. The predominantly inattentive subtype, on the other 
hand, is rare in this age group, and inattention symptoms become more apparent as the child grows older ; this may reflect either a true developmental pattern or/ and difficulty in measuring inattention in early childhood. Preschool ADHD persists into school age in 60$80 \%$ of cases [7].

\section{B. Symptom checklists and behavior rating scales}

Three basic types of assessment measures may be used in assessing ADHD:

1) "Broadband" measures of behavioral problems, including:

$>$ The Child Behavior Checklist (CBCL) [8].

$>$ The Behavioral Assessment Schedule for Children (BASC) [9].

D The Strengths and Difficulties Questionnaire (SDQ) [10].

All these exist in multiple forms by age and by informant (parent, teacher or child), none is specific to ADHD and they should not be used to diagnose ADHD or any other disorder. They can, however, be an appropriate first step in identifying co-morbidities [11].

2) Narrow-band questionnaires that are specific to the symptoms of ADHD

- Conners Rating Scale Revised (CRS-R) is most widely used, with versions available for parents, teachers and older children (as well as for adults) [12].

- Brown Attention Deficit Disorder Scale (BADDS) for children and adolescents [13].

- Vanderbilt ADHD Rating Scale [13].

- Swanson, Nolan, and Pelham IV (SNAP) [14].

- Childhood Attention Adjustment Survey (CAAS) [15].
3) Interview and other schedules that are based specifically on the DSM-V.

\subsection{Objective measures:}

\section{A. Neuropsychological measures}

- Specified batteries of neuropsychological tests have been proposed as a basis for diagnosis of ADHD, with particular emphasis on tests of executive functioning and working memory. A number of neuropsychological tests of attention and executive control, such as the Continuous Performance Task (CPT), have been investigated in the diagnosis of ADHD which is quite sensitive to central nervous system dysfunction. A number of disorders can result in impaired performance on the CPT. Reliance on the CPT as a primary diagnostic tool in determining the presence of ADHD could result in an unacceptably high number of false positives (i.e. over-diagnosis of ADHD). The CPT provides a quick standardized method of assessing attention and executive control. It shows promise in the monitoring of treatment response of those with ADHD; however, further research is needed on its utility in this area [16].

\section{B. Neurophysiological measures}

Electrophysiological measures such as event-related potentials (ERP) and electroencephalography (EEG) have been used to study brain processes in children with ADHD for more than 3 decades. EEG measures the electrical activity of the brain, allowing researchers to map the level of electrical activity occurring in certain brain regions.

The most consistent finding in the ADHD electrophysiological literature has been that clinical groups of children and adolescents with ADHD demonstrate increased low-frequency 
activity, primarily theta (or slow wave) power, when compared to healthy control groups. The increase in theta activity has also been reported in adults with ADHD [17].

It has been asserted in the literature that EEG measures may be used to differentiate between individuals with ADHD who will respond well to stimulant medication and non-responders. Children with ADHD who respond to medication have been reported to have specific EEG findings, in particular, excessive slow wave activity, which suggests that they are more cortically hypoaroused. Further research and validation are required to establish the utility of these findings [18].

\section{Neuroimaging}

The primary neuroimaging techniques are magnetic resonance imaging (MRI), functional magnetic resonance imaging (fMRI), diffusion tensor imaging (DTI), positron emission tomography (PET) and single photon emission computed tomography (SPECT). Structural neuroimaging (i.e. MRI) can be used to assess differences in brain structure, such as total brain volume or the size of specific regions of the brain. Functional neuroimaging techniques (fMRI, SPECT and PET) can also look at differences in regional cerebral blood flow [19].

Regionally, the most consistent theme that has emerged has been the repeated finding of the dorsal anterior mid-cingulate cortex (daMCC) dysfunction. The daMCC normally has key roles in attention, cognition, motor control/response selection, motivation, error detection, and feedback-based decision-making [20]. Numerous fMRI, PET, and ERP studies have reported daMCC hypofunction in ADHD, using various tasks and techniques [21]. Moreover, a metaanalysis of neuroimaging studies by Dickstein et al [22] found the daMCC among a short list of brain regions that were hypoactive in ADHD patients relative to healthy controls.

The research into the use of these techniques has been limited to differentiate between those with and without ADHD. Neuroimaging techniques are not enough to be used for diagnosis [23].

\section{Assessment of Attention Components}

In the presence of a growing demand for objective assessment measures, and because attention is a multicomponential concept, there is interest in measures that tap multiple components of attention. Heaton et al. [24] highlighted that being able to assess multiple components of attention to demonstrate both strengths and weaknesses is advantageous. Through assessment of multiple types of attention, significant impairment in one or more areas can be elucidated. There has been frequent agreement between researchers that sustained attention is impaired in children diagnosed with ADHD [25].

Since developmental trends in sustained attention exist in non-impaired children, there is interest in the ability to assess for deficits of sustained attention [26]. They noted that although a few sustained attention measures exist, usually in the form of some kind of objective continuous performance task requiring signal detection, more research on assessment of sustained attention in children is needed. Chan et al. [27] criticized the use of traditional continuous performance tasks, which provide information on only the sustained attention component, because children may not be 
able to employ the same engagement in these tasks as adults.

Manly [28] noted that continuous performance tasks are a unique contribution to assessment, but highlighted the potential advantage that could be gained by demonstrating shared variance on more than one sustained attention task in a battery, as opposed to one task alone. The Test of Everyday Attention for children (TEA-Ch) is one such battery that is able to provide assessment of performance related to multiple components of attention.

With the introduction of the TEA-Ch by Manly, et al., [6] a new opportunity in research on children's attention arrived in the field of attention assessment. The TEA-Ch is a nine subtests of performance tasks battery, which have received praise to children for having the appearance of games, that may rise effortful contribution in assessment [27]. TEA-Ch is one of the only objective assessment batteries available that is designed to measure more than one construct of attention while excluding measurement of other constructs such as verbal skills and executive functioning [24].

The TEA-Ch consists of nine subtests, each measuring one type of attention: selective, attention control/switching, and sustained attention. Utilizing a structural equation model of the TEA-Ch revealed a goodness of fit, where the latent variables (selective/focused attention, sustained attention, and attentional control) each linked to only one factor (subtests of the TEA-Ch) in the three-factor model [6].

The TEA-Ch appears to be sensitive to the measurement of sustained attention. Manly et al. [4] administered the TEA-Ch to 24 boys diagnosed with ADHD who had never been medicated with stimulants. The boys with ADHD performed significantly worse on sustained attention tasks compared to age and IQ matched controls.

Subsequently, several other researchers have demonstrated similar discriminative properties of the TEA-Ch among children with and without attention problems. In the first study using the TEA-Ch in the United States, Heaton et al. [24] noted that the TEA-Ch was able to significantly distinguish between a group of 63 children with ADHD and a non-ADHD group of 23 children on subtests of sustained attention and attentional switching/control. Hood et al. [29] found that when comparing a group of 15 children with ADHD with a group of 16 age, sex, and IQmatched non-ADHD children, the children with ADHD performed worse on subtests of sustained attention. In a similar study, Sutcliffe et al. [30] found that a group of 18 children with ADHD when compared to a group of 18 non-ADHD children performed significantly worse on subtests of sustained attention and attention switching/control. More recently, Chan et al. [27], in an attempt to validate translated versions of the TEA-Ch in China, demonstrated a significant impairment of performance on subtests of sustained attention with a clinical group of 22 children with ADHD in comparison to a group of 22 non-ADHD children. It appears that the subtests of the TEA-Ch have demonstrable ability to discriminate between children with and without ADHD. It follows then, that an objective measure of attention such as the TEA-Ch with potential to discriminate between clinical and non-clinical groups has power in predicting the presence or absence of an ADHD diagnosis. 


\subsection{Subjective versus Objective measures}

In a review of evidence-based assessment practices, Pelham et al. [5] recommended traditional behavior and symptom rating scales for clinical evaluation of ADHD because they are relatively efficient and cost effective for the clinician and client. However, these measures are subjective in nature because they rely on the opinion of a parent or teacher. They further suggested that lengthy structured clinical interviews, global assessments of impairment, and most observational assessments are not necessary to accurate diagnosis. In contrast to Pelham et al.'s position, Morein-Zamir et al. [31] questioned whether or not rating scales alone are enough to make informed diagnosis. Morein-Zamir et al.'s [31] position stems from their research which supports evidence of impairment of response inhibition in children with ADHD.

Manly et al. [4] expressed that rating scales may be the most useful diagnostic tool in demonstrating attention impairment, but their utility is limited as such measures do not demonstrate particular impairments. Because several factors associated with ADHD make diagnosing the disorder difficult (e.g., comorbid conditions, being older), and the sequelae of ADHD can have lasting implications [32], measures other than rating scales are sometimes necessary. In further support of the need for additional types of attention measures, Hood et al. [29] implied that cognitive assessment that includes objective measures of attention may be needed to detect more subtle forms of impairment in children with ADHD.

\section{Conclusion:}

Attention assessment includes subjective and objective measures. Subjective measures as behavior and symptom rating scales are the most useful diagnostic tool in demonstrating attention impairment, but that their value is limited in that such measures do not determine particular impairments. On the other hand, objective measures as continuous performance tasks are able to assess multiple types of attention and can detect more subtle forms of impairment in children with ADHD. So that, the use of both measures is beneficial to provide accurate diagnosis and treatment planning.

\section{References:}

[1] Trautmann, M., \& Zepf, F. D. (2012). Attentional Performance, Age and Scholastic Achievement in Healthy Children. PLoS ONE; 7(3): e32279.

[2] Rizzo, A., Buckwalter, J., Bowerly, T., van der Zaag, C., Humphrey, L., \& Neumann, U., (2000). The virtual classroom: A virtual reality environment for the assessment and rehabilitation of attention deficits. Cyber psychology \& Behavior; 3: 483-499

[3] American Psychiatry Association (2013). Diagnostic and statistical manual of mental disorders American Psychiatric Association. $5^{\text {th }}$ ed. Arlington: American Psychiatric Publishing; 5965.

[4] Manly, T., Nammo-Smith, I., Watson, P., Anderson, V., Turner, A., \& Robertson, I. H. (2001). The differential assessment of children's attention: The test of everyday attention for children (TEA-Ch), normative sample and ADHD performance. Journal of Child Psychology and Psychiatry; 42(8): 1065-1081. 
[5] Pelham, J. r., Fabiano, G. A., \& Massetti, G. M. (2005). Evidence-based assessment of attention deficit hyperactivity disorder in children and adolescents. Journal of Clinical Child \& Adolescent Psychology; 34(3): 449476.

[6] Manly, T., Robertson, I. H., Anderson, V., \& Nimmo-smith, I. (1999). TEA-Ch: The Test of Everyday Attention for Children Manual. Bury St. Edmunds, UK: Thames Valley Test Company Limited

[7] Galéra, C., Cote, S. M., Bouvard, M.P., Pingault, J. B., Melchior, M., \& Michel, G. (2011). Early risk factors for hyperactivity-impulsivity and inattention trajectories from age 17 months to 8 years. Arch General Psychiatry; 68(12): 1267-75.

[8] Achenbach, T.(1991). Manual for the Child Behavior Cheklist / 4-18 and 1991 profile. Burlington VT: University of Vermont. Department of psychiatry.

[9] Reynolds, C., \& Kamphaus, R. (1992): BASC: Behavior assessment system for children. Circle Pines, MN: American Guidance Service.

[10] Goodman, R. (1997): The Strengths and Difficulties Questionnaire: A Research Note. Journal of Child Psychology and Psychiatry; 38: 581-586.

[11] Biederman, J., Monuteaux, M. C., Kendrick, E., Klein, K. L. \& Faraone, S. V. (2005). The CBCL as a screen for psychiatric comorbidity in paediatric patients with ADHD. Arch Dis Child; 90: 1010-5.

[12] ] Collett, B. R., Ohan, J. L., \& Myers, K. M. (2003). Ten-year review of rating scales. V: scales assessing attentiondeficit/hyperactivity disorder. J Am
Acad Child Adolesc Psychiatry; 42:1015-37.

[13] Madaan, V., Daughton, J., Lubberstedt, B., Mattai, A., Vaughan, B. S., \& Kratochvil, C. J. (2008). Assessing the efficacy of treatments for ADHD: overview of methodological issues. J CNS Drugs; 22 (4): 275-90.

[14] Bussing, R., Fernandez, M., Harwood, M., Hou, W., Garvan, C. W., \& Eyberg, S. M. (2008). Parent and teacher SNAP-IV ratings of attention deficit hyperactivity disorder symptoms. Assessment; 15(3): 317-28.

[15] Lambert, N. M., Hartsough, C., \& Sandoval, J. (1990). Manual for the children's attention and adjustment survey. Chicago: Consulting Psychologists Press.

[16] National Health and Medical Research Council (2009). Australian guidelines on attention deficit hyperactivity disorder (ADHD).Australia: The Royal Australasian College of Physicians.

[17] Penberthy, J. K., Kalbfleisch, M. L., Quigg, M., Loboschefski, T., Cox, D., \& Runyon, C. (2006). Electroencephalographic profiles of children with symptoms of attention deficit hyperactivity disorder: a review of the literature. Curr Pediatr Rev; 2:17-32.

[18] Loo, S. K., \& Barkley, R. A. (2005). Clinical utility of EEG in attention deficit hyperactivity disorder. Appl Neuropsychol; 12:64-76.

[19] Bush, G., Valera, E. M., \& Seidman. L. J. (2005). Functional neuroimaging of attention- deficit/hyperactivity disorder: a review and suggested 
future directions. Biological

Psychiatry; 57:1273-84.

[20] Bush, G., Frazier, J. A., Rauch, S. L., Seidman, L. J., Whalen, P. J., Rosen, B. R., \& Biederman, J. (1999). Anterior cingulate cortex dysfunction in attentiondeficit/hyperactivity disorder revealed by fMRI and the counting stroop. Biological Psychiatry; 45: 1542-1552.

[21] Durston S., Davidson M. C., Mulder M. J., Spicer J. A., Galvan A., \& Tottenham N. (2007). Neural and behavioral correlates of expectancy violations in attention deficit hyperactivity disorder. J Child Psychol Psychiatry; 48: 881-889.

[22] Dickstein, S. G., Bannon, K., Xavier Castellanos, F., \& Milham, M. P. (2006). The neural correlates of attention deficit hyperactivity disorder: an ALE meta-analysis. J Child Psychol Psychiatry; 47: 1051-1062.

[27] Chan, R. C. K., Wang, L., Ye, J., Leung, W. W. Y., \& Mok, M. Y. K. (2008). A psychometric study of the Test of Everyday Attention for Children in the Chinese Setting. Archives of Clinical Neuropsychology; 23: 455-466

[28] Manly, T. (2005). Is there any value in the differential assessment of attention? British Journal of Developmental Psychology; 23(4): 507-512.

[29] Hood, J., Baird, G., Rankin, P. M., \& Isaacs, E. (2005). Immediate effects of methylphenidate on cognitive attention skills of children with attention-deficit- hyperactivity disorder. Developmental Medicine and Child Neurology; 47(6): 408-414.

[30] Sutcliffe, P.A., Bishop, D. V., \& Houghton, S. (2006). Sensitivity of four subtests
[23] Sadek, J. (2014): A clinical's Guide to ADHD (2nd ed.). Switzerland: Springer International Publishing.

[24] Heaton, C. C., Reader, S. K., Preston, A. S., Fennell, E. B., Puyana, O. E., Gill, N., \& Johnson, J. H. (2001). The Test of Everyday Attention for Children (TEA-Ch): Patterns of performance in children with ADHD and Clinical Controls. Child Neuropsychology; 7: 251-264

[25] Houghton, S. (2006). Advances in ADHD research through the lifespan: Common themes and implications. International Journal of Disability, Development and Education; 53: 263272.

[26] Betts, J., Mckay, J., Maruff, P., \& Anderson, V. (2006). The development of sustained attention in children: The effect of age and task load. Child Neuropsychology; 12: 205-221.

of the Test of Everyday Attention for Children (TEA-Ch) to stimulant medication in children with ADHD. Educational Psychology; 26(3): 325337.

[31] Morein-Zamir, S., Hommersen, P., Johnston, C. \& Kingstone, A. (2008). Novel measures of response performance and inhibition in children with ADHD. Journal of Abnormal Child Psychology; 36(8): 1199-1210.

[32] Barkley, R. A., Fischer, M., Smallish, L., \& Fletcher, K. (2006). The persistence of attention deficit/ hyperactivity disorder into young adulthood as a functioning of reporting source. Journal of Abnormal Psychology; 111 (2): 279-289. 